UDC 544.31

\author{
G.N. Shabanova, A.N. Korohodska, N.B. Deviatova
}

\title{
REFINEMENT OF THE SUBSOLIDUS STRUCTURE OF THE FOUR-COMPONENT SYSTEM $\mathrm{Fe}_{2} \mathrm{O}_{3}-\mathrm{CaO}-\mathrm{Al}_{2} \mathrm{O}_{3}-\mathrm{Cr}_{2} \mathrm{O}_{3}$
}

\begin{abstract}
National Technical University «Kharkiv Polytechnic Institute», Kharkiv, Ukraine
The article describes the structure of the ternary subsystems of the four-component system $\mathrm{CaO}-\mathrm{Al}_{2} \mathrm{O}_{3}-\mathrm{Fe}_{2} \mathrm{O}_{3}-\mathrm{Cr}_{2} \mathrm{O}_{3}$. The calculations were performed with regard to three-component compounds. A tetrahedron of the four-component system $\mathrm{CaO}-\mathrm{Al}_{2} \mathrm{O}_{3}-\mathrm{Fe}_{2} \mathrm{O}_{3}-\mathrm{Cr}_{2} \mathrm{O}_{3}$ has been plotted. The lengths of conodes and volumes of elementary tetrahedra were calculated. To study the relationship between elementary tetrahedra, a topological graph was constructed which allows using the obtained data in the study of oil-well cements based on this system. It has been established that the tetrahedron $\mathrm{CaCr}_{2} \mathrm{O}_{4}-\mathrm{CaFe}_{2} \mathrm{O}_{4}-$ $\mathrm{CaAl}_{2} \mathrm{O}_{4}-\mathrm{Ca}_{4} \mathrm{Al}_{2} \mathrm{Fe}_{2} \mathrm{O}_{10}$ has the least degree of asymmetry (1.87), excluding some tetrahedra with simple oxides. The phases forming this tetrahedron most likely exist in the $\mathrm{CaO}-\mathrm{Al}_{2} \mathrm{O}_{3}-\mathrm{Cr}_{2} \mathrm{O}_{3}-\mathrm{Fe}_{2} \mathrm{O}_{3}$ system. This will permit developing a sustainable process to fabricate oil-well cement materials based on the calcium aluminum-chromium cement without the use of special techniques to ensure high accuracy of dosing the initial components. In addition, when designing the compositions of binding materials, it is necessary to avoid the ternary compound localization area, because its coexistence with $\mathrm{CaO}$ will lead to the formation of portlandite in the cement clinker and to a significant decrease in the strength.
\end{abstract}

Keywords: oil-well cement; four-component system; geometric topological analysis; length of the conode; volume of elementary tetrahedral; topological graph; tetrahedra of the system.

DOI: $10.32434 / 0321-4095-2019-123-2-144-149$

\section{Introduction}

As shown by quite a long experience in drilling deep wells, solutions based on mineral binders are the most effective material to separate productive layers. The main requirements for cement grouting oil-well cements are as follows: (i) the solution must remain mobile throughout its transport into the annulus; and (ii), it should be low-permeable, durable and non-shrinkable after stopping the injection of the cement slurry. In addition, the cement stone should not change the properties under the impact of environment for a long time.

The use of oil-well cements for oil wells determines the need to study the different compositions of clinker. The application of the wastes of chemical industry can solve some problems [1]. The introduction of chromite and ferritic additives into the structure of cement clinker improves its physicochemical characteristics [2]. In this connection, it is of interest to study the system
$\mathrm{CaO}-\mathrm{Al}_{2} \mathrm{O}_{3}-\mathrm{Fe}_{2} \mathrm{O}_{3}-\mathrm{Cr}_{2} \mathrm{O}_{3}$ [3].

The four-component system $\mathrm{CaO}-\mathrm{Al}_{2} \mathrm{O}_{3}-$ $\mathrm{Fe}_{2} \mathrm{O}_{3}-\mathrm{Cr}_{2} \mathrm{O}_{3}$ has been described in detail elsewhere [4]. However, when splitting this system, the formation of the three-component $\mathrm{Ca}_{6} \mathrm{Al}_{4} \mathrm{Cr}_{2} \mathrm{O}_{15}$ compound in the $\mathrm{CaO}-\mathrm{Al}_{2} \mathrm{O}_{3}-\mathrm{Cr}_{2} \mathrm{O}_{3}$ system was not taken into account. This causes considerable difficulties in developing the compositions of cement materials based on aluminum-chromite binding materials. The study of this system will make it possible to predict their physical-mechanical and technical properties as well as its behavior under the conditions of service.

Earlier studies of the ternary subsystems, in which the four-component system were considered, allows triangulating them, taking into account all existing stable phases at a temperature of $1300^{\circ} \mathrm{C}$, and determining the geometric-topological characteristics of the phases of the subsystems. The investigation the structure of three-component 
systems in the field of subsolidus allowed us to consider further the sub-solidus structure of the fourcomponent system $\mathrm{CaO}-\mathrm{Al}_{2} \mathrm{O}_{3}-\mathrm{Fe}_{2} \mathrm{O}_{3}-\mathrm{Cr}_{2} \mathrm{O}_{3}$.

To establish stable conodes in this system, thermodynamic and geometric-topological methods of analysis were used. When splitting the $\mathrm{CaO}-$ $\mathrm{Al}_{2} \mathrm{O}_{3}-\mathrm{Fe}_{2} \mathrm{O}_{3}-\mathrm{Cr}_{2} \mathrm{O}_{3}$ concentration tetrahedron into elementary ones with the involvement of a geometrictopological analysis, it is possible to produce a unique closure of the faces with incident edges (conodes) through the common vertex into the elementary tetrahedron. The application of this method allows minimizing the number of necessary thermodynamic calculations.

Subsystem $\mathrm{CaO}-\mathrm{Al}_{2} \mathrm{O}_{3}-\mathrm{Fe}_{2} \mathrm{O}_{3}$ in the system $\mathrm{CaO}-\mathrm{Al}_{2} \mathrm{O}_{3}-\mathrm{Fe}_{2} \mathrm{O}_{3}-\mathrm{Cr}_{2} \mathrm{O}_{3}$ shows 12 stable binary phases and is divided into 12 elementary triangles. When studying the system in the region which is rich of $\mathrm{CaO}$, the existence of the ternary compound $\mathrm{Ca}_{4} \mathrm{Al}_{2} \mathrm{Fe}_{2} \mathrm{O}_{10}$, brownmillerite, should be taken into account [4]. The latter melts congruently at $1415^{\circ} \mathrm{C}$ having light refraction indices $\mathrm{Ng}=2.08, \mathrm{~Np}=1.98$ and forming a continuous series of solid solutions with $\mathrm{Ca}_{2} \mathrm{Fe}_{2} \mathrm{O}_{5}$.

In the $\mathrm{CaO}-\mathrm{Fe}_{2} \mathrm{O}_{3}-\mathrm{Cr}_{2} \mathrm{O}_{3}$ subsystem, ternary compounds are not formed. Oxides $\mathrm{Fe}_{2} \mathrm{O}_{3}$ and $\mathrm{Cr}_{2} \mathrm{O}_{3}$ form a series of solid solutions [5]. Considering the thermodynamic constants $\mathrm{CaCr}_{2} \mathrm{O}_{4}$, there is a $\mathrm{Fe}_{2} \mathrm{O}_{3}-\mathrm{CaCr}_{2} \mathrm{O}_{4}$ conode. Then, the other two conodes $\left(\mathrm{Ca}_{2} \mathrm{Fe}_{2} \mathrm{O}_{5}-\mathrm{CaCr}_{2} \mathrm{O}_{4}\right.$ and $\left.\mathrm{CaCr}_{2} \mathrm{O}_{4}-\mathrm{CaFe}_{4} \mathrm{O}_{7}\right)$ can be determined geometrically.

Ternary compounds are not formed in the subsystem $\mathrm{Fe}_{2} \mathrm{O}_{3}-\mathrm{Al}_{2} \mathrm{O}_{3}-\mathrm{Cr}_{2} \mathrm{O}_{3}$. There are continuous series of solid solutions at high temperatures in two binary systems $\left(\mathrm{Fe}_{2} \mathrm{O}_{3}-\mathrm{Cr}_{2} \mathrm{O}_{3}\right.$ and $\left.\mathrm{Cr}_{2} \mathrm{O}_{3}-\mathrm{Al}_{2} \mathrm{O}_{3}\right)$; this causes the formation of ternary solid solutions in a very vast area of the $\mathrm{Al}_{2} \mathrm{O}_{3}-\mathrm{Fe}_{2} \mathrm{O}_{3}-\mathrm{Cr}_{2} \mathrm{O}_{3}$ system. Their decay is observed only when the content of $\mathrm{Cr}_{2} \mathrm{O}_{3}$ is less than $30 \mathrm{wt} . \%$ and the temperature is about $1770 \mathrm{~K}$. With a decrease in the temperature, the region of ternary solutions diminishes. The presence of the only binary compound $\mathrm{FeAlO}_{3}$ causes the conditional formation of two elementary triangles $\mathrm{Cr}_{2} \mathrm{O}_{3}-\mathrm{Al}_{2} \mathrm{O}_{3}-\mathrm{FeAlO}_{3}$ and $\mathrm{Fe}_{2} \mathrm{O}_{3}-\mathrm{Cr}_{2} \mathrm{O}_{3}-\mathrm{FeAlO}_{3}$ $[7,8]$.

A three-component compound of the composition $\mathrm{Ca}_{6} \mathrm{Al}_{4} \mathrm{Cr}_{2} \mathrm{O}_{15}$ is formed in the subsystem $\mathrm{CaO}-\mathrm{Al}_{2} \mathrm{O}_{3}-\mathrm{Cr}_{2} \mathrm{O}_{3}$, which has a hexagonal structure with the following cell parameters: $\alpha_{0}=16.188 \AA$ and $\omega=14.864 \AA . \mathrm{Ca}_{6} \mathrm{Al}_{4} \mathrm{Cr}_{2} \mathrm{O}_{15}$ melts incongruently at $1641^{\circ} \mathrm{C}$ to form $\mathrm{CaCr}_{2} \mathrm{O}_{4}$ and a melt [5]. Under reducing conditions, the three-component phase, $\mathrm{Ca}_{6} \mathrm{Al}_{4} \mathrm{Cr}_{2} \mathrm{O}_{15}$, coexists with $\mathrm{CaO}, \mathrm{CaCr}_{2} \mathrm{O}_{4}$, chromitechromate and calcium aluminates. The chemical composition of the three-component compound $\mathrm{Ca}_{6} \mathrm{Al}_{4} \mathrm{Cr}_{2} \mathrm{O}_{15}$ can be given as follows: $\mathrm{CaO}-48.6 \%$, $\mathrm{Al}_{2} \mathrm{O}_{3}-29.4 \%$, and $\mathrm{Cr}_{2} \mathrm{O}_{3}-22 \%$ [4,8].

Combining the triangulated three-component subsystems $\mathrm{CaO}-\mathrm{Al}_{2} \mathrm{O}_{3}-\mathrm{Fe}_{2} \mathrm{O}_{3}, \mathrm{CaO}-\mathrm{Fe}_{2} \mathrm{O}_{3}-\mathrm{Cr}_{2} \mathrm{O}_{3}$, $\mathrm{CaO}-\mathrm{Al}_{2} \mathrm{O}_{3}-\mathrm{Cr}_{2} \mathrm{O}_{3}$ and $\mathrm{Al}_{2} \mathrm{O}_{3}-\mathrm{Fe}_{2} \mathrm{O}_{3}-\mathrm{Cr}_{2} \mathrm{O}_{3}$ into the concentration tetrahedron of the $\mathrm{CaO}-\mathrm{Al}_{2} \mathrm{O}_{3}-$ $\mathrm{Fe}_{2} \mathrm{O}_{3}-\mathrm{Cr}_{2} \mathrm{O}_{3}$ system allows determining its tetrahedra structure $[8,9]$.

\section{Results and discussion}

There are no four-component oxide phases in the four-component system under study; therefore it is advisable to begin from the top of $\mathrm{CaAl}_{2} \mathrm{O}_{4}$. The initial tetrahedron of the system $\mathrm{CaO}-\mathrm{Al}_{2} \mathrm{O}_{3}-\mathrm{Fe}_{2} \mathrm{O}_{3}-$ $\mathrm{Cr}_{2} \mathrm{O}_{3}$ is determined by the presence of the following tetrahedra:

1. $\mathrm{C}] \mathrm{aAl}{ }_{12} \mathrm{O}_{19}-\mathrm{Al}_{2} \mathrm{Fe}_{2} \mathrm{O}_{6}-\mathrm{Cr}_{2} \mathrm{O}_{3}-\mathrm{Al}_{2} \mathrm{O}_{3}$;

2. $\mathrm{CaAl}_{12} \mathrm{O}_{19}-\mathrm{Al}_{2} \mathrm{Fe}_{2} \mathrm{O}_{6}-\mathrm{Cr}_{2} \mathrm{O}_{3}-\mathrm{Fe}_{2} \mathrm{O}_{3}$;

3. $\mathrm{CaCr}_{2} \mathrm{O}_{4}-\mathrm{CaAl}_{12} \mathrm{O}_{19}-\mathrm{Cr}_{2} \mathrm{O}_{3}-\mathrm{Fe}_{2} \mathrm{O}_{3}$;

4. $\mathrm{CaCr}_{2} \mathrm{O}_{4}-\mathrm{CaAl}_{4} \mathrm{O}_{7}-\mathrm{CaAl}_{12} \mathrm{O}_{19}-\mathrm{Fe}_{2} \mathrm{O}_{3}$;

5. $\mathrm{CaCr}_{2} \mathrm{O}_{4}-\mathrm{CaAl}_{2} \mathrm{O}_{4}-\mathrm{CaFe}_{4} \mathrm{O}_{7}-\mathrm{Fe}_{2} \mathrm{O}_{3}$;

6. $\mathrm{CaCr}_{2} \mathrm{O}_{4}-\mathrm{CaAl}_{2} \mathrm{O}_{4}-\mathrm{CaAl}_{4} \mathrm{O}_{7}-\mathrm{CaFe}_{4} \mathrm{O}_{7}$;

7. $\mathrm{CaCr}_{2} \mathrm{O}_{4}-\mathrm{CaFe}_{2} \mathrm{O}_{4}-\mathrm{CaAl}_{2} \mathrm{O}_{4}-\mathrm{CaFe}_{4} \mathrm{O}_{7}$;

8. $\mathrm{CaCr}_{2} \mathrm{O}_{4}-\mathrm{CaFe}_{2} \mathrm{O}_{4}-\mathrm{Ca}_{2} \mathrm{Fe}_{2} \mathrm{O}_{5}-\mathrm{Ca}_{4} \mathrm{Al}_{2} \mathrm{Fe}_{2} \mathrm{O}_{10}$;

9. $\mathrm{CaCr}_{2} \mathrm{O}_{4}-\mathrm{CaFe}_{2} \mathrm{O}_{4}-\mathrm{CaAl}_{2} \mathrm{O}_{4}-\mathrm{Ca}_{4} \mathrm{Al}_{2} \mathrm{Fe}_{2} \mathrm{O}_{10}$;

10. $\mathrm{CaCr}_{2} \mathrm{O}_{4}-\mathrm{Ca}_{12} \mathrm{Al}_{14} \mathrm{O}_{33}-\mathrm{CaAl}_{2} \mathrm{O}_{4}-\mathrm{Ca}_{4} \mathrm{Al}_{2} \mathrm{Fe}_{2} \mathrm{O}_{10}$.

The presence of the three-component compound $\mathrm{Ca}_{6} \mathrm{Al}_{4} \mathrm{Cr}_{2} \mathrm{O}_{15}$ in the elementary triangle $\mathrm{CaCr}_{2} \mathrm{O}_{4}-\mathrm{Ca}_{3} \mathrm{Al}_{2} \mathrm{O}_{6}-\mathrm{Ca}_{12} \mathrm{Al}_{14} \mathrm{O}_{33}$ should be taken into account in geometrical-topological considerations of the internal connotation of $\mathrm{Ca}_{6} \mathrm{Al}_{4} \mathrm{Cr}_{2} \mathrm{O}_{15}-$ $\mathrm{Ca}_{4} \mathrm{Al}_{2} \mathrm{Fe}_{2} \mathrm{O}_{10}$, this causes the existence of the following elementary tetrahedra:

11. $\mathrm{CaCr}_{2} \mathrm{O}_{4}-\mathrm{Ca}_{4} \mathrm{Al}_{2} \mathrm{Fe}_{2} \mathrm{O}_{10}-\mathrm{Ca}_{12} \mathrm{Al}_{14} \mathrm{O}_{33}-\mathrm{Ca}_{6} \mathrm{Al}_{4} \mathrm{Cr}_{2} \mathrm{O}_{15}$; 12. $\mathrm{CaO}-\mathrm{Ca}_{4} \mathrm{Al}_{2} \mathrm{Fe}_{2} \mathrm{O}_{10}-\mathrm{Ca}_{6} \mathrm{Al}_{4} \mathrm{Cr}_{2} \mathrm{O}_{15}-\mathrm{CaCr}_{2} \mathrm{O}_{4}$;

13. $\mathrm{Ca}_{3} \mathrm{Al}_{2} \mathrm{O}_{6}-\mathrm{Ca}_{4} \mathrm{Al}_{2} \mathrm{Fe}_{2} \mathrm{O}_{10}-\mathrm{CaO}-\mathrm{Ca}_{6} \mathrm{Al}_{4} \mathrm{Cr}_{2} \mathrm{O}_{15}$;

14. $\mathrm{Ca}_{6} \mathrm{Al}_{4} \mathrm{Cr}_{2} \mathrm{O}_{15}-\mathrm{Ca}_{3} \mathrm{Al}_{2} \mathrm{O}_{6}-\mathrm{Ca}_{12} \mathrm{Al}_{14} \mathrm{O}_{33}-\mathrm{Ca}_{4} \mathrm{Al}_{2} \mathrm{Fe}_{2} \mathrm{O}_{10}$; 15. $\left.\mathrm{CaO}-\mathrm{Ca}_{2} \mathrm{Fe}_{2} \mathrm{O}_{5}-\mathrm{Ca}_{4} \mathrm{~A}\right] \mathrm{l}_{2} \mathrm{Fe}_{2} \mathrm{O}_{10}-\mathrm{CaCr}_{2} \mathrm{O}_{4}$.

The tetrahedral system of $\mathrm{Fe}_{2} \mathrm{O}_{3}-\mathrm{CaO}-\mathrm{Al}_{2} \mathrm{O}_{3}-$ $\mathrm{Cr}_{2} \mathrm{O}_{3}$ with allowance for the stable phases is shown in Fig. 1.

Thus, when splitting the concentration tetrahedron into elementary ones, two «internal» connections were established which pass the threedimensional space of the concentration tetrahedron: $\mathrm{Ca}_{6} \mathrm{Al}_{4} \mathrm{Cr}_{2} \mathrm{O}_{15}-\mathrm{Ca}_{4} \mathrm{Al}_{2} \mathrm{Fe}_{2} \mathrm{O}_{10}$ and $\mathrm{Ca}_{4} \mathrm{Al}_{2} \mathrm{Fe}_{2} \mathrm{O}_{10}-$ $\mathrm{CaCr}_{2} \mathrm{O}_{4}$; they determine the presence of 15 elementary tetrahedra in the subsolidus region.

The lengths of the conodes and the volumes of elementary tetrahedra were calculated taking into 


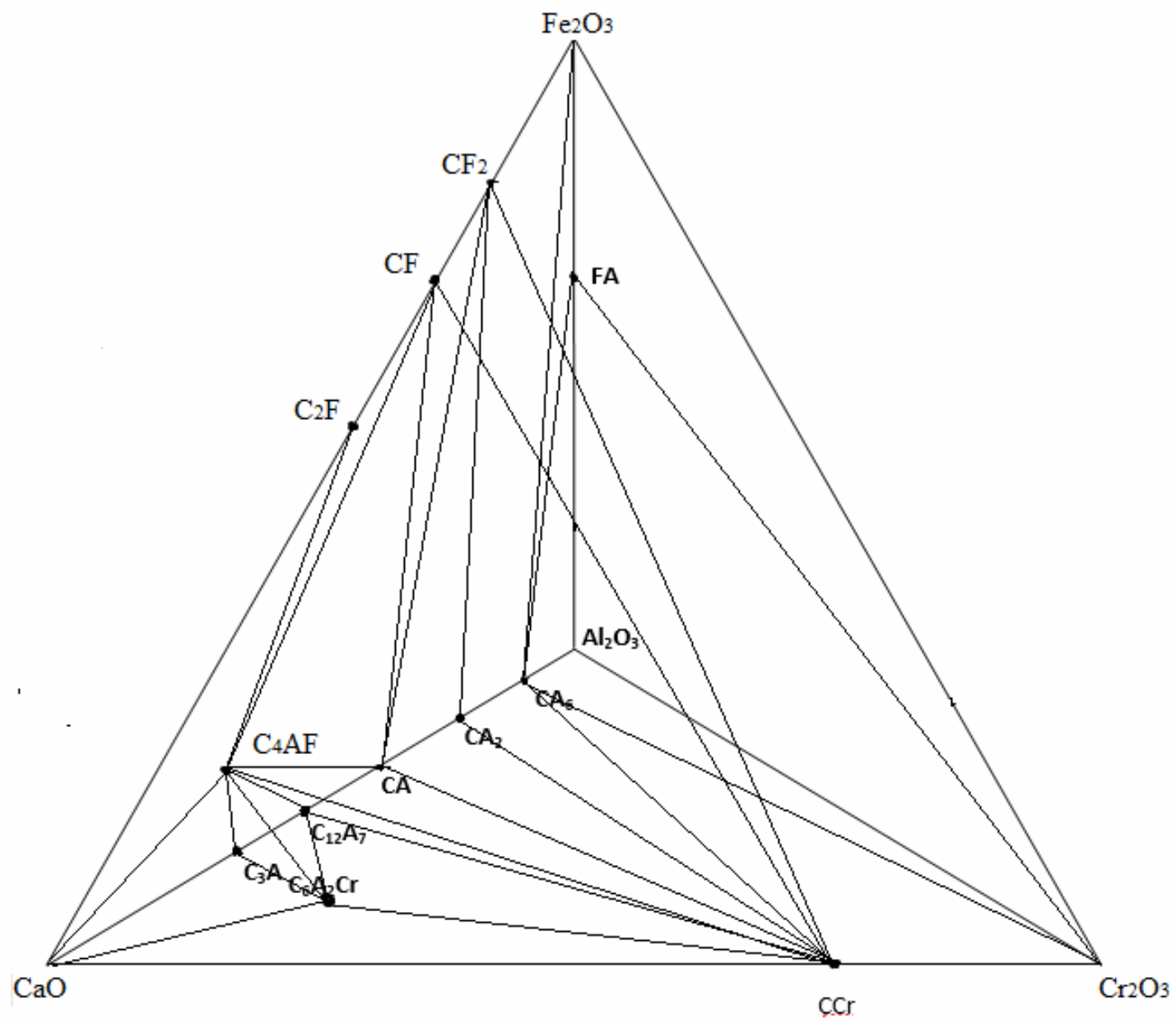

Fig. 1. Tetrahedron system $\mathrm{Fe}_{2} \mathrm{O}_{3}-\mathrm{CaO}-\mathrm{Al}_{2} \mathrm{O}_{3}-\mathrm{Cr}_{2} \mathrm{O}_{3}$ with regard to stable phases

account barycentric coordinates and elements of Euclidean geometry, the results of calculations are presented in Table 1.

To study the relationship of elementary tetrahedra, a topological graph was constructed (Fig. 2), for which, using the Euler formula [4], the number of edges was calculated to be 16 .

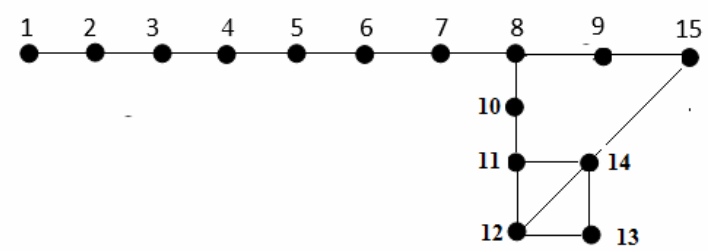

Fig. 2. Topological graph of interconnection of elementary tetrahedra of the system $\mathrm{CaO}-\mathrm{Al}_{2} \mathrm{O}_{3}-\mathrm{Fe}_{2} \mathrm{O}_{3}-\mathrm{Cr}_{2} \mathrm{O}_{3}$

There are two combinations of phases 4 and 7 in the system, which not immediately follow from the three-component subsystems constituting it. There are no an «insertion» tetrahedra in the system in which none of the faces comes out on the surface of the concentration tetrahedron $\mathrm{CaO}-\mathrm{Al}_{2} \mathrm{O}_{3}-$ $\mathrm{Cr}_{2} \mathrm{O}_{3}-\mathrm{Fe}_{2} \mathrm{O}_{3}$. There is one hanging point in the graph that corresponds to the elementary tetrahedron no. 1 , in which three of four faces face the surface of the concentration tetrahedron of the system. The graph is flat, with no «false» intersections of the ribs.

As can be seen from the presented results (Tables 2 and 3), the following tetrahedra have the largest relative volumes: $\mathrm{CaCr}_{2} \mathrm{O}_{4}-\mathrm{CaAl}_{12} \mathrm{O}_{19}-\mathrm{Cr}_{2} \mathrm{O}_{3}-$ $\mathrm{Fe}_{2} \mathrm{O}_{3}, \mathrm{CaCr}_{2} \mathrm{O}_{4}-\mathrm{CaAl}_{4} \mathrm{O}_{7}-\mathrm{CaAl}_{12} \mathrm{O}_{19}-\mathrm{Fe}_{2} \mathrm{O}_{3}$, $\mathrm{CaCr}_{2} \mathrm{O}_{4}-\mathrm{CaFe}_{2} \mathrm{O}_{4}-\mathrm{CaAl}_{2} \mathrm{O}_{4}-\mathrm{Ca}_{4} \mathrm{Al}_{2} \mathrm{Fe}_{2} \mathrm{O}_{10}$ and $\mathrm{CaCr}_{2} \mathrm{O}_{4}-\mathrm{CaAl}_{2} \mathrm{O}_{4}-\mathrm{CaAl}_{4} \mathrm{O}_{7}-\mathrm{CaFe}_{4} \mathrm{O}_{7}$ (0.2469; $0.0961,0.0805$, and 0.0864 relative units, respectively). Excluding tetrahedra with simple oxides, the tetrahedron $\mathrm{CaCr}_{2} \mathrm{O}_{4}-\mathrm{CaFe}_{2} \mathrm{O}_{4}-$ $\mathrm{CaAl}_{2} \mathrm{O}_{4}-\mathrm{Ca}_{4} \mathrm{Al}_{2} \mathrm{Fe}_{2} \mathrm{O}_{10}$ has the least degree of asymmetry (1.87). The phases that form this tetrahedron most likely exist in the $\mathrm{CaO}-\mathrm{Al}_{2} \mathrm{O}_{3}-$ $\mathrm{Cr}_{2} \mathrm{O}_{3}-\mathrm{Fe}_{2} \mathrm{O}_{3}$ system (Table 3), which will allow developing a sustainable technology to create cement 
Characteristics of conode system $\mathrm{CaO}-\mathrm{Al}_{2} \mathrm{O}_{3}-\mathrm{Cr}_{2} \mathrm{O}_{3}-\mathrm{Fe}_{2} \mathrm{O}_{3}$

\begin{tabular}{|c|c|}
\hline Phase & Coexisting phases and conode length, $\%$ \\
\hline $\mathrm{Al}_{2} \mathrm{O}_{3}$ & $\mathrm{CaO}(1.0) ; \mathrm{Fe}_{2} \mathrm{O}_{3}(1.0) ; \mathrm{Cr}_{2} \mathrm{O}_{3}(1.0)$ \\
\hline $\mathrm{CaO}$ & $\begin{array}{l}\mathrm{Ca}_{2} \mathrm{Fe}_{2} \mathrm{O}_{5}(0.5874) ; \mathrm{Ca}_{4} \mathrm{Al}_{2} \mathrm{Fe}_{2} \mathrm{O}_{10}(0.470) ; \mathrm{Ca}_{3} \mathrm{Al}_{2} \mathrm{O}_{6}(0.3474) ; \mathrm{Ca}_{6} \mathrm{Al}_{4} \mathrm{Cr}_{2} \mathrm{O}_{15}(0.446672) ; \mathrm{CaCr}_{2} \mathrm{O}_{4} \\
(0.443801)\end{array}$ \\
\hline $\mathrm{Fe}_{2} \mathrm{O}_{3}$ & $\begin{array}{l}\mathrm{Cr}_{2} \mathrm{O}_{3}(1.0) ; \mathrm{CaCr}_{2} \mathrm{O}_{4}(0.896175) ; \mathrm{Al}_{2} \mathrm{Fe}_{2} \mathrm{O}_{6}(0.3897) ; \mathrm{CaAl}_{12} \mathrm{O}_{19}(0.960771) ; \mathrm{CaAl}_{4} \mathrm{O}_{7}(0.911497) ; \\
\mathrm{CaFe}_{4} \mathrm{O}_{7}(0.1494)\end{array}$ \\
\hline $\mathrm{Cr}_{2} \mathrm{O}_{3}$ & $\mathrm{Fe}_{2} \mathrm{O}_{3}(1.0) ; \mathrm{Al}_{2} \mathrm{Fe}_{2} \mathrm{O}_{6}(0.873021) ; \mathrm{Al}_{2} \mathrm{O}_{3}(1,0) ; \mathrm{CaAl}_{12} \mathrm{O}_{19}(0.960771) ; \mathrm{CaCr}_{2} \mathrm{O}_{4}(0.2695)$ \\
\hline $\mathrm{CaCr}_{2} \mathrm{O}_{4}$ & $\begin{array}{l}\mathrm{CaAl}_{12} \mathrm{O}_{19}(0.838798) ; \mathrm{CaAl}_{4} \mathrm{O}_{7}(0.758832) ; \mathrm{CaAl}_{2} \mathrm{O}_{4}(0.691805) ; \mathrm{Ca}_{12} \mathrm{Al}_{14} \mathrm{O}_{33}(0.650045) ; \\
\mathrm{Ca}_{6} \mathrm{Al}_{4} \mathrm{Cr}_{2} \mathrm{O}_{15}(0.443801) ; \mathrm{CaO}(0.7305) ; \mathrm{Cr}_{2} \mathrm{O}_{3}(0.2695) ; \mathrm{Fe}_{2} \mathrm{O}_{3}(0.896175) ; \mathrm{CaFe}_{2} \mathrm{O}_{4}(0.735347) ; \\
\mathrm{Ca}_{2} \mathrm{Fe}_{2} \mathrm{O}_{5}(0.670502) ; \mathrm{CaFe}_{4} \mathrm{O}_{7}(0.797363)\end{array}$ \\
\hline $\mathrm{CaAl}_{12} \mathrm{O}_{19}$ & $\begin{array}{l}\mathrm{Al}_{2} \mathrm{O}_{3}(0.08397) ; \mathrm{CaCr}_{2} \mathrm{O}_{4}(0.838798) ; \mathrm{Cr}_{2} \mathrm{O}_{3}(0.960771) ; \mathrm{CaAl}_{4} \mathrm{O}_{7}(0.13173) ; \mathrm{Fe}_{2} \mathrm{O}_{3}(0.960771) ; \\
\mathrm{Al}_{2} \mathrm{Fe}_{2} \mathrm{O}_{6}(0.3897)\end{array}$ \\
\hline $\mathrm{CaAl}_{4} \mathrm{O}_{7}$ & $\mathrm{CaAl}_{12} \mathrm{O}_{19}(0.13173) ; \mathrm{CaCr}_{2} \mathrm{O}_{4}(0.758832) ; \mathrm{CaAl}_{2} \mathrm{O}_{4}(0.1391) ; \mathrm{Fe}_{2} \mathrm{O}_{3}(0.911497) ; \mathrm{CaFe}_{4} \mathrm{O}_{7}(0.819464)$ \\
\hline $\mathrm{CaAl}_{2} \mathrm{O}_{4}$ & $\begin{array}{l}\mathrm{CaAl}_{4} \mathrm{O}_{7}(0.1391) ; \mathrm{CaCr}_{2} \mathrm{O}_{4}(0.691805) ; \mathrm{Ca}_{12} \mathrm{Al}_{14} \mathrm{O}_{33}(0.1305) ; \mathrm{CaFe}_{4} \mathrm{O}_{7}(0.819464) ; \mathrm{CaFe}_{2} \mathrm{O}_{4} \\
(0.735347) ; \mathrm{Ca}_{4} \mathrm{Al}_{2} \mathrm{Fe}_{2} \mathrm{O}_{10}(0.393038)\end{array}$ \\
\hline $\mathrm{Ca}_{12} \mathrm{Al}_{14} \mathrm{O}_{33}$ & $\begin{array}{l}\mathrm{CaAl}_{2} \mathrm{O}_{4}(0.1305) ; \mathrm{CaCr}_{2} \mathrm{O}_{4}(0.650045) ; \mathrm{Ca}_{6} \mathrm{Al}_{4} \mathrm{Cr}_{2} \mathrm{O}_{15}(0.220351) ; \mathrm{Ca}_{3} \mathrm{Al}_{2} \mathrm{O}_{6}(0.1373) ; \mathrm{Ca}_{4} \mathrm{Al}_{2} \mathrm{Fe}_{2} \mathrm{O}_{10} \\
(0.317414)\end{array}$ \\
\hline $\mathrm{Ca}_{3} \mathrm{Al}_{2} \mathrm{O}_{6}$ & $\mathrm{Ca}_{12} \mathrm{Al}_{14} \mathrm{O}_{33}(0.1373) ; \mathrm{Ca}_{6} \mathrm{Al}_{4} \mathrm{Cr}_{2} \mathrm{O}_{15}(0.192373) ; \mathrm{CaO}$ (0.3774); $\mathrm{Ca}_{4} \mathrm{Al}_{2} \mathrm{Fe}_{2} \mathrm{O}_{10}(0.284595)$ \\
\hline $\mathrm{Ca}_{6} \mathrm{Al}_{4} \mathrm{Cr}_{2} \mathrm{O}_{15}$ & $\mathrm{CaO}(0.446672) ; \mathrm{Ca}_{3} \mathrm{Al}_{2} \mathrm{O}_{6}(0.192373) ; \mathrm{Ca}_{12} \mathrm{Al}_{14} \mathrm{O}_{33}(0.220351) ; \mathrm{CaCr}_{2} \mathrm{O}_{4}(0.443801)$ \\
\hline $\mathrm{Ca}_{4} \mathrm{Al}_{2} \mathrm{Fe}_{2} \mathrm{O}_{10}$ & $\begin{array}{l}\mathrm{CaO}(0.470036) ; \mathrm{CaAl}_{2} \mathrm{O}_{4}(0.393038) ; \mathrm{Ca}_{12} \mathrm{Al}_{14} \mathrm{O}_{33}(0.317414) ; \mathrm{Ca}_{3} \mathrm{Al}_{2} \mathrm{O}_{6}(0.284595) ; \mathrm{CaFe}_{2} \mathrm{O}_{4} \\
(0.356392) ; \mathrm{Ca}_{2} \mathrm{Fe}_{2} \mathrm{O}_{5}(0238112)\end{array}$ \\
\hline $\mathrm{Al}_{2} \mathrm{Fe}_{2} \mathrm{O}_{6}$ & $\mathrm{Fe}_{2} \mathrm{O}_{3}(0.3897) ; \mathrm{Cr}_{2} \mathrm{O}_{3}(0.873021) ; \mathrm{Al}_{2} \mathrm{O}_{3}(0.6103) ; \mathrm{CaAl}_{12} \mathrm{O}_{19}(0.572949)$ \\
\hline $\mathrm{CaFe}_{2} \mathrm{O}_{4}$ & $\begin{array}{l}\mathrm{Ca}_{2} \mathrm{Fe}_{2} \mathrm{O}_{5}(0.1527) ; \mathrm{CaFe}_{4} \mathrm{O}_{7}(0.1105) ; \mathrm{CaCr}_{2} \mathrm{O}_{4}(0.735347) ; \mathrm{CaAl}_{2} \mathrm{O}_{4}(0.697509) ; \mathrm{Ca}_{4} \mathrm{Al}_{2} \mathrm{Fe}_{2} \mathrm{O}_{10} \\
(0.356392) ;\end{array}$ \\
\hline $\mathrm{CaFe}_{4} \mathrm{O}_{7}$ & $\mathrm{Fe}_{2} \mathrm{O}_{3}(0.1494) ; \mathrm{CaFe}_{2} \mathrm{O}_{4}(0.1105) ; \mathrm{CaCr}_{2} \mathrm{O}_{4}(0.797363) ; \mathrm{CaAl}_{4} \mathrm{O}_{7}(0.819464) ; \mathrm{CaAl}_{2} \mathrm{O}_{4}(0.768763)$ \\
\hline $\mathrm{Ca}_{2} \mathrm{Fe}_{2} \mathrm{O}_{5}$ & $\mathrm{CaFe}_{2} \mathrm{O}_{4}(0.1527) ; \mathrm{CaCr}_{2} \mathrm{O}_{4}(0.670502) ; \mathrm{CaO}(0.5874) ; \mathrm{Ca}_{4} \mathrm{Al}_{2} \mathrm{Fe}_{2} \mathrm{O}_{10}(0.238112)$ \\
\hline
\end{tabular}

Characterization of elementary tetrahedra of the system $\mathrm{CaO}-\mathrm{Al}_{2} \mathrm{O}_{3}-\mathrm{Cr}_{2} \mathrm{O}_{3}-\mathrm{Fe}_{2} \mathrm{O}_{3}$

\begin{tabular}{|c|c|c|c|}
\hline No. & Elementary tetrahedron & Volume, relative units & Degree of asymmetry \\
\hline 1 & $\mathrm{CaAl}_{12} \mathrm{O}_{19}-\mathrm{Al}_{2} \mathrm{Fe}_{2} \mathrm{O}_{6}-\mathrm{Cr}_{2} \mathrm{O}_{3}-\mathrm{Al}_{2} \mathrm{O}_{3}$ & 0.0512 & 11.909 \\
\hline 2 & $\mathrm{CaAl}_{12} \mathrm{O}_{19}-\mathrm{Al}_{2} \mathrm{Fe}_{2} \mathrm{O}_{6}-\mathrm{Cr}_{2} \mathrm{O}_{3}-\mathrm{Fe}_{2} \mathrm{O}_{3}$ & 0.0330 & 1.7453 \\
\hline 3 & $\mathrm{CaCr}_{2} \mathrm{O}_{4}-\mathrm{CaAl}_{12} \mathrm{O}_{19}-\mathrm{Cr}_{2} \mathrm{O}_{3}-\mathrm{Fe}_{2} \mathrm{O}_{3}$ & 0.2469 & 1.1922 \\
\hline 4 & $\mathrm{CaCr}_{2} \mathrm{O}_{4}-\mathrm{CaAl}_{4} \mathrm{O}_{7}-\mathrm{CaAl}_{12} \mathrm{O}_{19}-\mathrm{Fe}_{2} \mathrm{O}_{3}$ & 0.0961 & 7.2935 \\
\hline 5 & $\mathrm{CaCr}_{2} \mathrm{O}_{4}-\mathrm{CaAl}_{2} \mathrm{O}_{4}-\mathrm{CaFe}_{4} \mathrm{O}_{7}-\mathrm{Fe}_{2} \mathrm{O}_{3}$ & 0.0856 & 5.9985 \\
\hline 6 & $\mathrm{CaCr}_{2} \mathrm{O}_{4}-\mathrm{CaAl}_{2} \mathrm{O}_{4}-\mathrm{CaAl}_{4} \mathrm{O}_{7}-\mathrm{CaFe}_{4} \mathrm{O}_{7}$ & 0.0864 & 5.8912 \\
\hline 7 & $\mathrm{CaCr}_{2} \mathrm{O}_{4}-\mathrm{CaFe}_{2} \mathrm{O}_{4}-\mathrm{CaAl}_{2} \mathrm{O}_{4}-\mathrm{CaFe}_{4} \mathrm{O}_{7}$ & 0.0521 & 1.1432 \\
\hline 8 & $\mathrm{CaCr}_{2} \mathrm{O}_{4}-\mathrm{CaFe}_{2} \mathrm{O}_{4}-\mathrm{Ca}_{2} \mathrm{Fe}_{2} \mathrm{O}_{5}-\mathrm{Ca}_{4} \mathrm{Al}_{2} \mathrm{Fe}_{2} \mathrm{O}_{10}$ & 0.0234 & 6.6547 \\
\hline 9 & $\mathrm{CaCr}_{2} \mathrm{O}_{4}-\mathrm{CaFe}_{2} \mathrm{O}_{4}-\mathrm{CaAl}_{2} \mathrm{O}_{4}-\mathrm{Ca}_{4} \mathrm{Al}_{2} \mathrm{Fe}_{2} \mathrm{O}_{10}$ & 0.0805 & 1.8709 \\
\hline 10 & $\mathrm{CaCr}_{2} \mathrm{O}_{4}-\mathrm{Ca}_{12} \mathrm{Al}_{14} \mathrm{O}_{33}-\mathrm{CaAl}_{2} \mathrm{O}_{4}-\mathrm{Ca}_{4} \mathrm{Al}_{2} \mathrm{Fe}_{2} \mathrm{O}_{10}$ & 0.0312 & 4.9812 \\
\hline 11 & $\mathrm{CaCr}_{2} \mathrm{O}_{4}-\mathrm{Ca}_{4} \mathrm{Al}_{2} \mathrm{Fe}_{2} \mathrm{O}_{10}-\mathrm{Ca}_{12} \mathrm{Al}_{14} \mathrm{O}_{33}-\mathrm{Ca}_{6} \mathrm{Al}_{4} \mathrm{Cr}_{2} \mathrm{O}_{15}$ & 0.0158 & 4.9941 \\
\hline 12 & $\mathrm{CaO}-\mathrm{Ca}_{4} \mathrm{Al}_{2} \mathrm{Fe}_{2} \mathrm{O}_{10}-\mathrm{Ca}_{6} \mathrm{Al}_{4} \mathrm{Cr}_{2} \mathrm{O}_{15}-\mathrm{CaCr}_{2} \mathrm{O}_{4}$ & 0.0706 & 2.5505 \\
\hline 13 & $\mathrm{Ca}_{3} \mathrm{Al}_{2} \mathrm{O}_{6}-\mathrm{Ca}_{4} \mathrm{Al}_{2} \mathrm{Fe}_{2} \mathrm{O}_{10}-\mathrm{CaO}-\mathrm{Ca}_{6} \mathrm{Al}_{4} \mathrm{Cr}_{2} \mathrm{O}_{15}$ & 0.0273 & 2.4433 \\
\hline 14 & $\mathrm{Ca}_{6} \mathrm{Al}_{4} \mathrm{Cr}_{2} \mathrm{O}_{15}-\mathrm{Ca}_{3} \mathrm{Al}_{2} \mathrm{O}_{6}-\mathrm{Ca}_{12} \mathrm{Al}_{14} \mathrm{O}_{33}-\mathrm{Ca}_{4} \mathrm{Al}_{2} \mathrm{Fe}_{2} \mathrm{O}_{10}$ & 0.0099 & 2.3210 \\
\hline 15 & $\mathrm{CaO}-\mathrm{Ca}_{2} \mathrm{Fe}_{2} \mathrm{O}_{5}-\mathrm{Ca}_{4} \mathrm{Al}_{2} \mathrm{Fe}_{2} \mathrm{O}_{10}-\mathrm{CaCr}_{2} \mathrm{O}_{4}$ & 0.090 & 3.0679 \\
\hline & Amount & 1.0 & - \\
\hline & Max & 0.2469 & 11.909 \\
\hline & Min & 0.0099 & 1.1432 \\
\hline
\end{tabular}


Geometrical-topological characteristics of the phases in system $\mathrm{CaO}-\mathrm{Al}_{2} \mathrm{O}_{3}-\mathrm{Cr}_{2} \mathrm{O}_{3}-\mathrm{Fe}_{2} \mathrm{O}_{3}$

\begin{tabular}{l|c|c|c|c}
\hline \multicolumn{1}{c|}{ Compound } & $\begin{array}{c}\text { How many } \\
\text { phases coexist }\end{array}$ & $\begin{array}{c}\text { How many } \\
\text { tetrahedra exist }\end{array}$ & $\begin{array}{c}\text { Scope of existence, } \mathrm{V}_{\mathrm{i}}, \\
\text { relative units }\end{array}$ & $\begin{array}{c}\text { Probability of existence } \omega, \\
\text { relative units }\end{array}$ \\
\hline $\mathrm{Al}_{2} \mathrm{O}_{3}$ & 3 & 1 & 0.0512 & 0.0128 \\
\hline $\mathrm{Cr}_{2} \mathrm{O}_{3}$ & 5 & 3 & 0.3311 & 0.0828 \\
\hline $\mathrm{CaO}$ & 5 & 3 & 0.1870 & 0.0468 \\
\hline $\mathrm{Fe}_{2} \mathrm{O}_{3}$ & 6 & 4 & 0.4610 & 0.1153 \\
\hline $\mathrm{CaCr}_{2} \mathrm{O}_{4}$ & 11 & 11 & 0.8770 & 0.2192 \\
\hline $\mathrm{CaAl}_{12} \mathrm{O}_{19}$ & 6 & 4 & 0.4372 & 0.1093 \\
\hline $\mathrm{CaAl}_{4} \mathrm{O}_{7}$ & 5 & 3 & 0.2681 & 0.067 \\
\hline $\mathrm{CaAl}_{2} \mathrm{O}_{4}$ & 6 & 4 & 0.2472 & 0.0618 \\
\hline $\mathrm{Ca}_{3} \mathrm{Al}_{2} \mathrm{O}_{6}$ & 4 & 2 & 0.0372 & 0.0093 \\
\hline $\mathrm{Ca}_{12} \mathrm{Al}_{14} \mathrm{O}_{33}$ & 5 & 3 & 0.0563 & 0.0141 \\
\hline $\mathrm{Ca}_{6} \mathrm{Al}_{4} \mathrm{Cr}_{2} \mathrm{O}_{15}$ & 4 & 4 & 0.1230 & 0.0308 \\
\hline $\mathrm{Al}_{2} \mathrm{Fe}_{2} \mathrm{O}_{6}$ & 4 & 2 & 0.0832 & 0.0208 \\
\hline $\mathrm{CaFe}_{2} \mathrm{O}_{4}$ & 5 & 3 & 0.155 & 0.0387 \\
\hline $\mathrm{CaFe}_{4} \mathrm{O}_{7}$ & 4 & 3 & 0.2241 & 0.0560 \\
\hline $\mathrm{Ca}_{2} \mathrm{Fe}_{2} \mathrm{O}_{5}$ & 4 & 2 & 0.1134 & 0.0283 \\
\hline $\mathrm{Ca}_{4} \mathrm{Al}_{2} \mathrm{Fe}_{2} \mathrm{O}_{10}$ & 6 & 8 & 0.3480 & 0.0870 \\
\hline $\mathrm{Amount}$ & - & - & 4.0000 & 1.0000 \\
\hline $\mathrm{Max}_{\mathrm{Min}}$ & 7 & 6 & 0.8770 & 0.2192 \\
\hline
\end{tabular}

materials based on calcium aluminum chromium cement without special techniques for ensuring high accuracy of dosing the initial components. In addition, when designing compositions of binding materials, it is necessary to avoid the ternary compound localization area, since its coexistence with $\mathrm{CaO}$ will lead to the formation of portlandite in cement clinker and a significant decrease in strength.

\section{Conclusions}

We described the construction of triple subsystems of the four-component system $\mathrm{CaO}-$ $\mathrm{Al}_{2} \mathrm{O}_{3}-\mathrm{Fe}_{2} \mathrm{O}_{3}-\mathrm{Cr}_{2} \mathrm{O}_{3}$. The tetrahedra of the fourcomponent system $\mathrm{CaO}-\mathrm{Al}_{2} \mathrm{O}_{3}-\mathrm{Fe}_{2} \mathrm{O}_{3}-\mathrm{Cr}_{2} \mathrm{O}_{3}$ were plotted and discussed. The parameters of the elemental tetrahedra were calculated. A topological graph showing the interrelations between elementary tetrahedra was constructed. The obtained results can be used to study self-grouting cements based on this system.

\section{REFERENCES}

1. Ridha S., Irawan S., Ariwahjoedi B. Prediction equation for permeability of class $G$ Oilwell cement under reservoir conditions // Arabian J. Sci. Eng. - 2014. - Vol.39. - No. 6. -
P.5219-5228.

2. Odler I. Special inorganic cements. - New York: CRC Press, 2000. $-390 \mathrm{p}$.

3. Song S., Garbers-Craig A.M. Formation, leachability and encapsulation of hexavalent chromium in the $\mathrm{Al}_{2} \mathrm{O}_{3}-\mathrm{CaO}-$ $\mathrm{Fe}_{2} \mathrm{O}_{3}-\mathrm{Cr}_{2} \mathrm{O}_{3}$ system // J. Europ. Ceram. Soc. -2016 . - Vol.36. - P.1479-1485.

4. Berezhnoj A.S. Mnogokomponentnye sistemy okislov. Kiev: Naukova Dumka, 1970. -544 p.

5. Kaiser A., Sommer B., Woermann E. The system $\mathrm{CaO}-" \mathrm{CaCr}_{2} \mathrm{O}_{4} "-\mathrm{CaAl}_{2} \mathrm{O}_{4}$ in air and under mildly reducing conditions // J. Am. Ceram. Soc. - 1992. - Vol.75. - No. 161. - P.1463-71.

6. Shabanova G.N., Korogodskaja A.N., Deviatova N.B. Utochnenie subsolidusnogo stroeniya sistemy $\mathrm{CaO}-\mathrm{Al}_{2} \mathrm{O}_{3}-\mathrm{Cr}_{2} \mathrm{O}_{3}$ $\mathrm{s}$ uchetom troinogo soedineniya $\mathrm{Ca}_{6} \mathrm{Al}_{4} \mathrm{Cr}_{2} \mathrm{O}_{15} / / \mathrm{J}$. Refract. Tech. Ceram. - 2017. - No. 9. - P.18-23.

7. Formation and leachability of hexavalent chromium in the $\mathrm{Al}_{2} \mathrm{O}_{3}-\mathrm{CaO}-\mathrm{MgO}-\mathrm{Cr}_{2} \mathrm{O}_{3}$ system / Wu Y., Song S., GarbersCraig A.M., Xue Z. // J. Europ. Ceram. Soc. - 2018. - Vol.38. - No. 6. - P.2649-2661.

8. Teilor H. Khimiya tsementa. - Moscow: Mir, 1996. $560 \mathrm{p}$.

9. Kurdowski W. Cement and concrete chemistry. Springer Netherlands. -2014 . - 700 p.

Received 16.11.2018 


\section{УТОЧНЕННЯ СУБСОЛІДУСНОЇ БУДОВИ ЧОТИРИКОМПОНЕНТНОЇ СИСТЕМИ $\mathrm{Fe}_{2} \mathrm{O}_{3}-\mathrm{CaO}-\mathrm{Al}_{2} \mathrm{O}_{3}-\mathrm{Cr}_{2} \mathrm{O}_{3}$}

\section{Г.М. Шабанова, А.М. Корогодська, Н.Б. Дев'ятова}

У статті розглянуті будова потрійних підсистем чотирикомпонентної системи $\mathrm{CaO}-\mathrm{Al}_{2} \mathrm{O}_{3}-\mathrm{Fe}_{2} \mathrm{O}_{3}-\mathrm{Cr}_{2} \mathrm{O}_{3}$. Додані розрахунки з урахуванням трикомпонентних з'єднань. Здійснена тетраедрація чотирикомпонентної системи $\mathrm{CaO}-\mathrm{Al}_{2} \mathrm{O}_{3}-$ $\mathrm{Fe}_{2} \mathrm{O}_{3}-\mathrm{Cr}_{2} \mathrm{O}_{3}$. Розраховані довжини коннод і обсяги елементарних тетраедрів. Для вивчення взаємозв'язку елементарних тетраедрів побудований топологічний граф, що дозволило використовувати отримані дані при вивченні властивостей тампонажних иементів на основі цієї системи. Встановлено що, виключаючи тетраєдри з простими оксидами, найменший ступінь асиметрії (1,87) має тетраедр $\mathrm{CaCr}_{2} \mathrm{O}_{4}-\mathrm{CaFe}_{2} \mathrm{O}_{4}-$ $\mathrm{CaAl}_{2} \mathrm{O}_{4}-\mathrm{Ca}_{4} \mathrm{Al}_{2} \mathrm{Fe}_{2} \mathrm{O}_{10}$. Для фаз, які входять до складу даного тетраедра, притаманна найбільша ймовірність існування в системі $\mathrm{CaO}-\mathrm{Al}_{2} \mathrm{O}_{3}-\mathrm{Cr}_{2} \mathrm{O}_{3}-\mathrm{Fe}_{2} \mathrm{O}_{3}$, що дозволить розробити стійку технологію створення тампонажних матеріалів на основі кальцієвого алюмохромітного цементу без спеціальних прийомів щодо забезпечення високої точності дозування вихідних компонентів. Також при проектуванні складів в яжучих матеріалів необхідно уникати області локалізації потрійного з'єднання, оскільки його співіснування з СаО буде призводити до утворення портландіта в цементному клінкері $i$ значного зниження міцності.

Ключові слова: тампонажний цемент, чотирикомпонентна система, геометро-топологічний аналіз, довжини коннод, обсяг елементарних тетраедрів, топологічний граф, тетраедрація системи.

\section{REFINEMENT OF THE SUBSOLIDUS STRUCTURE OF THE FOUR-COMPONENT SYSTEM Fe $\mathrm{O}_{3}-\mathrm{CaO}-\mathrm{Al}_{2} \mathrm{O}_{3}-\mathrm{Cr}_{2} \mathrm{O}_{3}$}

\section{G.N. Shabanova *, A.N. Korohodska, N.B. Deviatova}

National Technical University «Kharkiv Polytechnic Institute», Kharkiv, Ukraine

\section{*e-mail: gala-shabanova@ukr.net}

The article describes the structure of the ternary subsystems of the four-component system $\mathrm{CaO}-\mathrm{Al}_{2} \mathrm{O}_{3}-\mathrm{Fe}_{2} \mathrm{O}_{3}-\mathrm{Cr}_{2} \mathrm{O}_{3}$. The calculations were performed with regard to three-component compounds. A tetrahedron of the four-component system $\mathrm{CaO}-\mathrm{Al}_{2} \mathrm{O}_{3}-$ $\mathrm{Fe}_{2} \mathrm{O}_{3}-\mathrm{Cr}_{2} \mathrm{O}_{3}$ has been plotted. The lengths of conodes and volumes of elementary tetrahedra were calculated. To study the relationship between elementary tetrahedra, a topological graph was constructed which allows using the obtained data in the study of oil-well cements based on this system. It has been established that the tetrahedron $\mathrm{CaCr}_{2} \mathrm{O}_{4}-\mathrm{CaFe}_{2} \mathrm{O}_{4}-\mathrm{CaAl}_{2} \mathrm{O}_{4}-\mathrm{Ca}_{4} \mathrm{Al}_{2} \mathrm{Fe}_{2} \mathrm{O}_{10}$ has the least degree of asymmetry (1.87), excluding some tetrahedra with simple oxides. The phases forming this tetrahedron most likely exist in the $\mathrm{CaO}-$ $\mathrm{Al}_{2} \mathrm{O}_{3}-\mathrm{Cr}_{2} \mathrm{O}_{3}-\mathrm{Fe}_{2} \mathrm{O}_{3}$ system. This will permit developing a sustainable process to fabricate oil-well cement materials based on the calcium aluminum-chromium cement without the use of special techniques to ensure high accuracy of dosing the initial components. In addition, when designing the compositions of binding materials, it is necessary to avoid the ternary compound localization area, because its coexistence with $\mathrm{CaO}$ will lead to the formation of portlandite in the cement clinker and to a significant decrease in the strength.

Keywords: oil-well cement; four-component system; geometric topological analysis; length of the conode; volume of elementary tetrahedral; topological graph; tetrahedra of the system.

\section{REFERENCES}

1. Ridha S., Irawan S., Ariwahjoedi B. Prediction equation for permeability of class G Oilwell cement under reservoir conditions. Arabian Journal for Science and Engineering, 2014, vol. 39 , pp. 5219-5228.

2. Odler I., Special inorganic cements. CRC Press, 2000.

3. Song S., Garbers-Craig A.M. Formation, leachability and encapsulation of hexavalent chromium in the $\mathrm{Al}_{2} \mathrm{O}_{3}-\mathrm{CaO}-$ $\mathrm{Fe}_{2} \mathrm{O}_{3}-\mathrm{Cr}_{2} \mathrm{O}_{3}$ system. Journal of the European Ceramic Society, 2016, vol. 36, pp. 1479-1485.

4. Berezhnoj A.S., Mnogokomponentnye sistemy okislov [Multicomponent oxide systems]. Naukova Dumka, Kiev, 1970. 544 p. (in Russian).

5. Kaiser A., Sommer B., Woermann E. The system $\mathrm{CaO}-" \mathrm{CaCr}_{2} \mathrm{O}_{4} "-\mathrm{CaAl}_{2} \mathrm{O}_{4}$ in air and under mildly reducing conditions. Journal of the American Ceramic Society, 1992, vol. 75 , pp. 1463-1471.

6. Shabanova G.N., Korogodskaja A.N., Deviatova N.B. Utochnenie subsolidusnogo stroeniya sistemy $\mathrm{CaO}-\mathrm{Al}_{2} \mathrm{O}_{3}-\mathrm{Cr}_{2} \mathrm{O}_{3} \mathrm{~S}$ uchetom troinogo soedineniya $\mathrm{Ca}_{6} \mathrm{Al}_{4} \mathrm{Cr}_{2} \mathrm{O}_{15}$ [Refinement of the subsolidus structure of the $\mathrm{CaO}-\mathrm{Al}_{2} \mathrm{O}_{3}-\mathrm{Cr}_{2} \mathrm{O}_{3}$ system with regard to the ternary compound $\mathrm{Ca}_{6} \mathrm{Al}_{4} \mathrm{Cr}_{2} \mathrm{O}_{15}$. Journal of the Refractories and Technical Ceramics, 2017, no. 9, pp.18-23. (in Russian).

7. Wu Y., Song S., Garbers-Craig A.M., Xue Z. Formation and leachability of hexavalent chromium in the $\mathrm{Al}_{2} \mathrm{O}_{3}-\mathrm{CaO}-$ $\mathrm{MgO}-\mathrm{Cr}_{2} \mathrm{O}_{3}$ system. Journal of the European Ceramic Society, 2018, vol. 38, pp. 2649-2661.

8. Teilor H., Khimiya tsementa [Cement chemistry]. Mir, Moscow, 1996. 560 p. (in Russian).

9. Kurdowski W., Cement and concrete chemistry. Springer Netherlands, 2014. $700 \mathrm{p}$. 